\title{
Natriuretic peptides regulate sympathetic nervous activity independent of mineralocorticoid receptor
}

\author{
Hitoshi Nakagawa*, Yasuki Nakada, Yoshihiko Saito \\ From 7th International Conference on cGMP Generators, Effectors and Therapeutic Implications \\ Trier, Germany. 19-21 June 2015
}

\begin{abstract}
Background
Natriuretic peptides (ANP/BNP) increase cGMP and exert cardiovascular protective effects via guanylyl cyclase A (GC-A) receptor, which is distributed in many organs such as the heart, the vasculature and the brain [1]. Sympathetic nervous system (SNS) as well as reninangiotensin-aldosterone-system contributes to cardiovascular disease. However, the endogenous effect of GC-A signaling on SNS is not investigated. Recent study shows that activated mineralocorticoid receptor (MR) in the hypothalamus induces systemic SNS activation [2], whereas ANP infusion in human inhibited SNS activity in the heart [3]. Notably, it is reported that ANP counteracts the deleterious effects of MR in the heart [4]. Therefore, we hypothesized that ANP suppresses MR activation in the brain and leads to the inhibition of SNS activity.
\end{abstract}

\section{Purpose}

To investigate whether ANP/GC-A signaling inhibits SNS activity through the suppression of the brain MR, we examined urinary catecholamine secretion in global GC-A receptor $\mathrm{KO}$ mice and the effect of intracerebroventricular (ICV) infusion of MR blocker.

\section{Methods and results}

We measured blood pressure (BP) and urinary norepinephrine (U-NE) secretion in wild type and global GC-A $\mathrm{KO}$ mice. Both BP and U-NE is significantly higher in GC-A KO than in wild type mice, indicating SNS is activated in GC-A KO mice. To study whether SNS activation is caused by the brain MR, we infused Eplerenone (MR blocker) into the ICV with osmotic mini pump for 2 weeks. Contrary to our hypothesis, both BP and U-NE did not change after 2 weeks ICV infusion, suggesting that activated SNS in GC-A KO is independent of MR. Furthermore, high sodium diet $(\mathrm{NaCl} 6 \%)$ for 2 weeks did not increase BP and U-NE in GC-A KO mice. MR protein expression in the hypothalamus was almost similar between GC-A KO and Wild type mice. These data suggest that SNS activity in GC-A KO mice is independent of MR and insensitive to sodium load. Unexpectedly, the most of GC-A KO mice died after ICV infusion of Losartan (AT1 receptor blocker), whereas wild type mice survived.

\section{Conclusion}

Natriuretic peptides/GC-A signaling regulates SNS activity independent of both brain MR and sodium load. Brain AT1 receptor might be important in the regulation of cardiovascular system in global GC-A KO mice.

Published: 2 September 2015

\section{References}

1. Kuhn M: Structure, regulation, and function of mammalian membrane guanylyl cyclase receptors, with a focus on guanylyl cyclase-A. Circ Res 2003, 93(8):700-709.

2. Hamlyn JM, Blaustein MP: Salt sensitivity, endogenous ouabain and hypertension. Curr Opin Nephrol Hypertens 2013, 22(1):51-58.

3. Kasama S, Toyama T, Kumakura H, Takayama Y, Ishikawa T, Ichikawa S, et al: Effects of intravenous atrial natriuretic peptide on cardiac sympathetic nerve activity in patients with decompensated congestive heart failure. J Nucl Med 2004, 45(7):1108-1113.

4. Nakagawa H, Oberwinkler H, Nikolaev VO, Gaßner B, Umbenhauer S, Wagner $H$, et al: Atrial natriuretic peptide locally counteracts the deleterious effects of cardiomyocyte mineralocorticoid receptor activation. Circ Heart Fail 2014, 7(5):814-821.

doi:10.1186/2050-6511-16-S1-A71

Cite this article as: Nakagawa et al.: Natriuretic peptides regulate sympathetic nervous activity independent of mineralocorticoid receptor. BMC Pharmacology and Toxicology 2015 16(Suppl 1):A71.

\footnotetext{
* Correspondence: nhitoshi@mub.biglobe.ne.jp

First Department of Internal Medicine, Nara Medical University, Kashihara, Japan
} 